J. of the Chosun Natural Science

Vol. 6, No. 2 (2013) pp. $118-123$

http://dx.doi.org/10.13160/ricns.2013.6.2.118

\title{
A Study of Comparison of Temperature Seasons and Biotic Seasons in Jeonnam Region
}

\author{
Sung Eun Hwang and Chan Su Ryu ${ }^{\dagger}$
}

\begin{abstract}
Korea, which is located in the middle-latitude area of the northern hemisphere, has four seasons. These seasons can be classified based on their months, mean temperatures, biology and natural phenomena. In this study, climatic changes were identified by biotic and temperature seasons, changing trends were compared by season, and the appropriateness of the biotic phenomena for the biotic season classification was examined. The data for this study included the mean temperatures, and biology · phenomenon observation dates, which had been observed for 38 years from the ASOSs in seven Gwangju and Jeonnam regions. Limitations were found in the classification of the temperature and biotic seasons. Especially in the case of the biotic season based on a single life, the points of the first sighting and the initial sound fluctuated so much that the accuracy of the results was not guaranteed. Therefore, the life species had to be selected subject to detailed verification and accurate specifications, and to be applied to the meteorological phenomena. In addition, there were lives in the standard biology that could no longer be observed because of environmental pollution and climatic change, which indicates the need to protect the existing standard biology.
\end{abstract}

Key words: Climate Change, Temperature Seasons, Biotic Seasons

\section{Introduction}

Korea, which is located in the middle-latitude area of the northern hemisphere, has four seasons. These seasons can be classified based on their months, mean temperatures, and biology $\cdot$ natural phenomena ${ }^{[1,2]}$. The classification by month is a statistical method that divides a year into four periods: spring (March to May), summer (June to August), fall (September to November) and winter (December to February). This method does not consider climatic changes and actual temperature changes ${ }^{[3,4]}$. Although this method is inaccurate, it is widely used because it is comprehensive. The standard of the National Institute of Meteorological Research

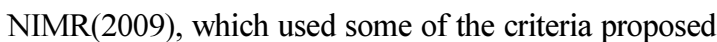
by Lee Byeongseol (1979), is a classification based on the mean temperature. This method is limited because it uses a single climatic element: the mean temperature. To supplement this limitation, the Korea Meteorological

Department of Atmospheric science, Chosun University, Gwangju 501759, Korea

†Corresponding author : csryu@chosun.ac.kr

(Received: May 14, 2013, Revised : June 20, 2013,

Accepted : June 24, 2013)
Administration $^{[5]}$ examines the biotic season data by observing representative lives and natural phenomena for each season. The biotic season is determined by observing the seasonal changes with reference to living things such as flowers and animals. This classification uses diverse climatic elements such as the temperature, isolation duration, amount of precipitation, and humidity, which change according to the seasonal progress ${ }^{[6,7]}$.

In this study, the climatic changes were identified by biotic and temperature seasons, the changing trends were compared by season, and the appropriateness of the biotic phenomena for the biotic season classification was examined.

\section{Experimental Section}

\subsection{Data and Analysis Method}

The data that were used in this study were the mean temperatures, and biology ·phenomenon observation dates observed from the ASOSs of seven Gwangju and Jeonnam regions (Mokpo, Yeosu, Wando, Suncheon, Jangheung, Goheung and Haenam). The climatological observation data that were used were for 38 years - from 1973, when the latest ASOS in Suncheon was installed, 
to $2010^{[3]}$. The observation date was indicated as a Julian day, with January 1 represented as 1 and December 31 , as 365 . The temperature seasons were classified according to the NIMR standard (2009) ${ }^{[8,9,10]}$. According to this standard, spring starts on the day with a daily mean temperature of $5^{\circ} \mathrm{C}$ or higher; summer, on the day with a daily mean temperature of $20^{\circ} \mathrm{C}$ or higher; fall, on the day with a daily mean temperature of $20^{\circ} \mathrm{C}$ or lower; and winter, on the day with a daily mean temperature of $5^{\circ} \mathrm{C}$ or lower.

The nine-day consecutive mean was selected as the mean temperature to filter the effects of the abnomal climate and significant weather. Each season was determined when the seasonal criterion was met for five consecutive days. For the biotic season, five criteria forsythia, cicada, cosmos, dragonfly and ice - were analyzed. The significance test for the temperature and biotic seasons was conducted using mainly the biotic season for each season, and a Kendall- $\tau$ correlation analysis was also conducted.

$$
\tau=\frac{S}{\sqrt{\frac{N^{2}-N-\tau_{x}}{2} \sqrt{\frac{N^{2}-N-\tau_{y}}{2}}}}
$$

\section{Results and Discussion}

\subsection{Temperature Season}

Figure 1 shows the changes in the mean temperature from 1973 to 1980 (in the 1970s) and from 2001 to 2010 (in the 2000s). The mean temperature was higher in the 2000s than in the 1970s in all the seasons, which

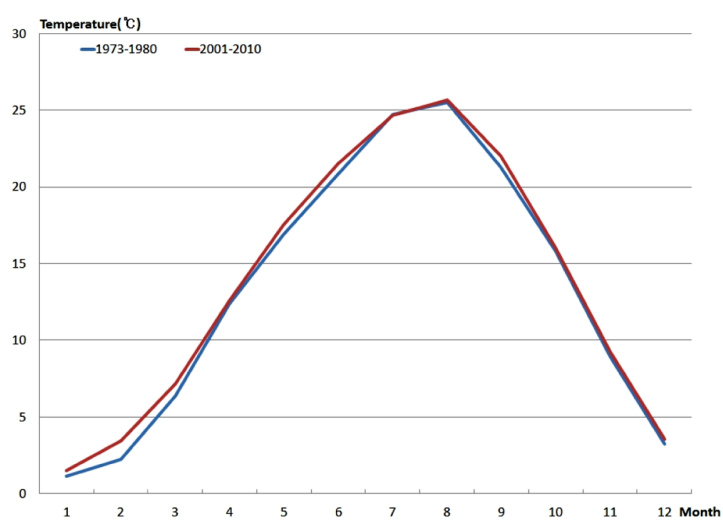

Fig. 1. Annual variation in the mean temperature from 1973 to 1980 and from 2001 to 2010.

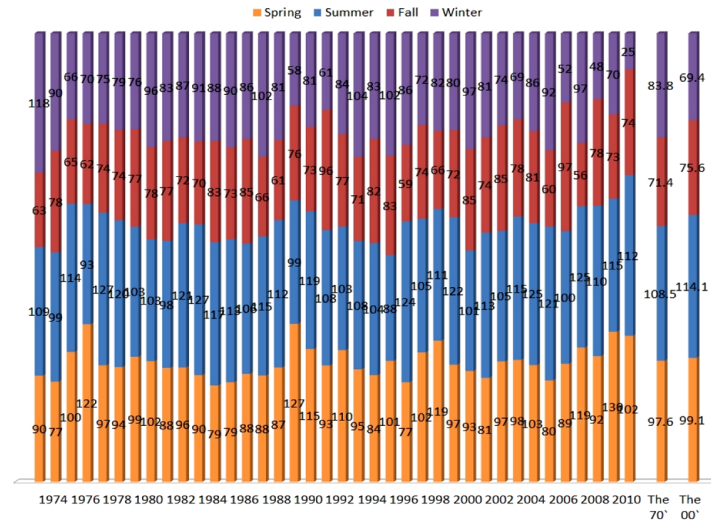

Fig. 2. Interannual variation in the starting date of the temperature season. In the length of the temperature season and comparison of the 1970s and the 2000s.

indicates a temperature increase. The highest temperature difference between the two periods appeared in February, which was $1.2^{\circ} \mathrm{C}$, and there was no temperature difference in July.

Figure 2 shows the length of each season, which was measured using the temperature season. Although no significant change was observed because of El Nio and La Nia, spring became 1.5 days longer, from 97.6 days in the 1970 s to 99.1 days in the 2000 s; summer, 5.6 days longer, from 108.5 days to 114.1 days; and fall, 4.2 days longer, from 71.4 days to 75.6 days; whereas winter became 9.4 days shorter, from 83.8 days to 74.3 days. Winter became significantly shorter, and summer had the greatest increase in number of days, followed by fall and spring. These changes would be more distinct if the effects of El Nino and La Niña were removed.

Figure 3 shows the starting dates of the temperature season for the past 38 years (1973-2010). Spring started on March 3 in the 1970s, but seven days earlier, on February 25, in the 2000s. Summer started on June 9 in the 1970s, but six days earlier, on June 3, in the 2000s. Fall started on September 25 in the 1970s, and on almost the same date, September 24, in the 2000s. Finally, winter started on

December 5 in the 1970s, but four days later, on December 9, in the 2000s. Winter started later and spring started earlier, which indicate that winter became shorter. Summer started earlier without a significant change in the starting date of fall, which indicates that summer became longer. Winter started later without a 


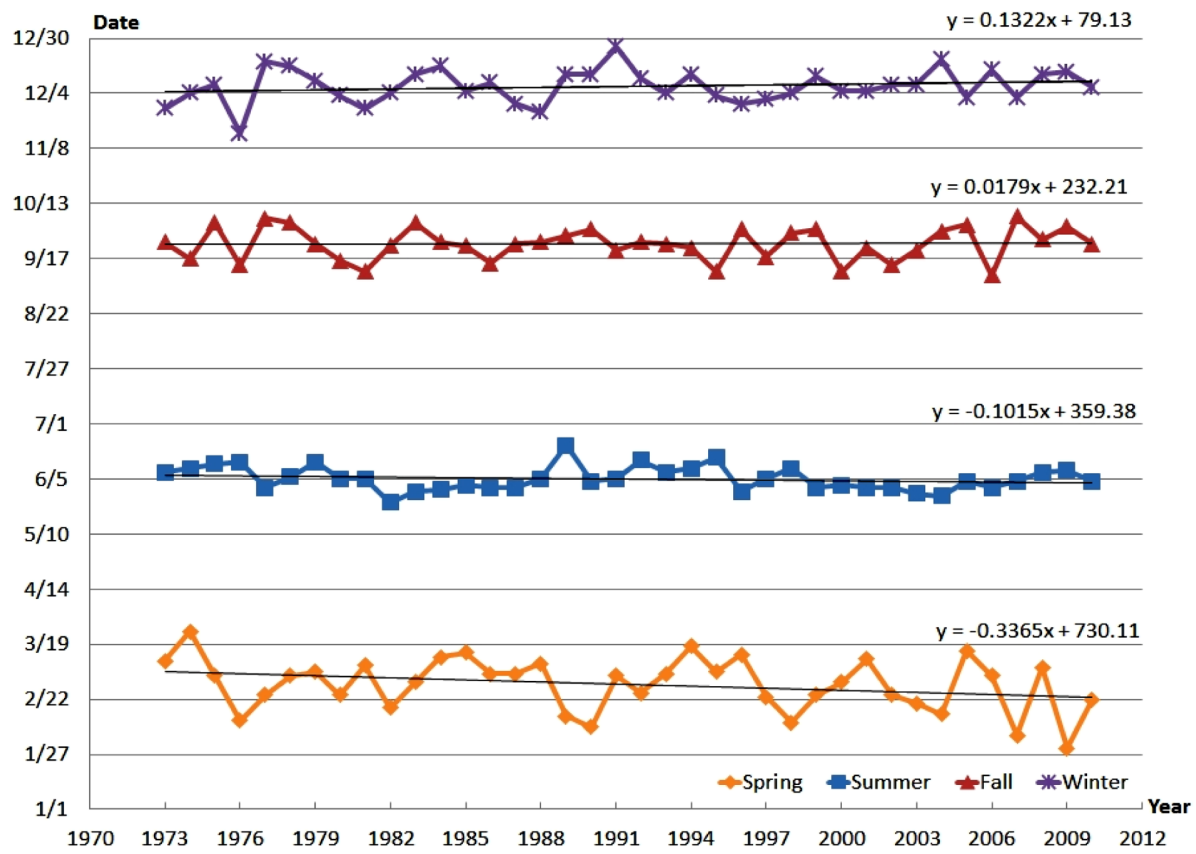

Fig. 3. Interannual variation in the starting date of the temperature season.

significant change in the starting date of fall, which indicates that fall became longer. As for the changing trend of the starting dates of the seasons, spring and summer started early at a rate of 0.132 day/year and 0.018 day/year, respectively, and fall and winter started late at a rate of -0.102 day/year and -0.337 day/year, respectively. The change was highest in winter and lowest in summer. In the trend and starting date analysis results, the summer period became longer because the starting date of fall was delayed, and the winter period became shorter because the starting date of winter was delayed and spring started early.

\subsection{Biotic Season}

Life and natural phenomena naturally represent the seasons. Accordingly, KMA designated standard plants such as forsythia, cherry blossoms and cosmos, and standard bugs such as cicada and dragonfly, to observe the start and end of the seasons, and considers the first sighting of ice as the start of winter, considering that water freezes at $0^{\circ} \mathrm{C}$ In this section, the start of a season was analyzed based on biological phenomena, and its relationship with the starting date of the temperature season was analyzed. Figure 4 shows the secular changes in the observation point of the first sighting (initial sound) of biological phenomena.

\subsubsection{Forsythia}

Forsythia can be easily seen across Korea in spring, and has been designated as the standard plant for spring in the country ${ }^{[11]}$. Therefore, the starting date of spring was estimated using the inflorescence date of forsythia. The mean forsythia inflorescence date for the past 38 years was March 22. It became four days earlier, from March 23 in the 1970s to March 19 in the 2000s, unlike the temperature season, which became 20 days earlier in the 1970s and 23 days earlier in the 2000s. It was 22 days earlier on the average than the spring of the temperature season. Both the forsythia inflorescence date and the starting date of the temperature season were early, and the Kendall- $\tau$ correlation analysis resulted in a correlation coefficient of 0.55 and a significance level of 0.01 .

\subsubsection{Cicada}

The cicada is a standard bug that represents summer, and starts to sing as summer starts ${ }^{[10]}$. Therefore, the initial sound of the cicada was taken as the indication of the starting point of summer. The mean initial cicada sound date for the past 38 years was July 11 . The date 


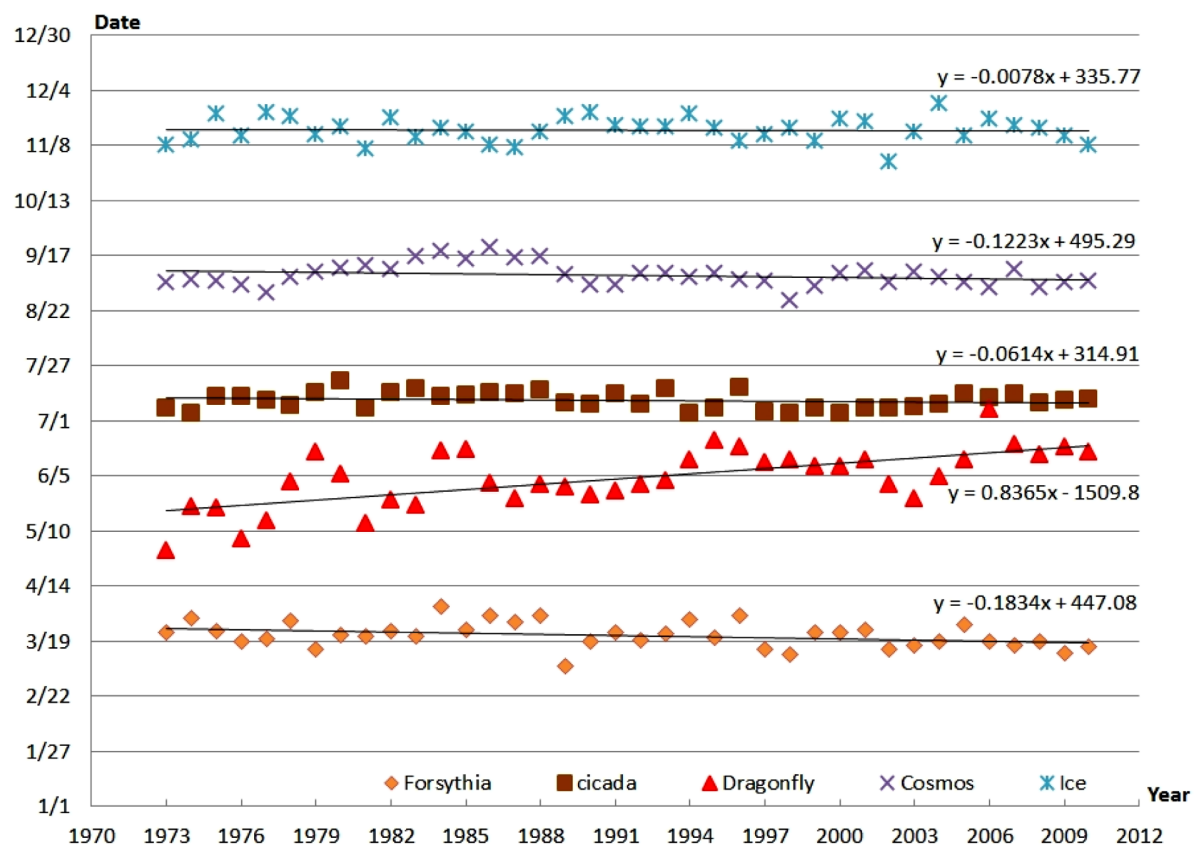

Fig. 4. Interannual variation in the observation days of the biotic season.

was July 11 and July 10 in the 1970s and the 2000s, respectively, which are almost the same. Compared with the starting date of the temperature season, the date was 32 days earlier in the 1970 s and 37 days earlier in the 2000 s, and 36 days earlier on the average. The initial cicada sound date was almost constant, but the starting date of the temperature season became six days early. Besides, the Kendall- $\tau$ correlation analysis results showed no coherence between the two elements.

\subsubsection{Cosmos}

The cosmos is grown worldwide for ornamental purposes, and is a standard fall plant that blossoms from June to October. Accordingly, the starting date of fall was estimated according to the cosmos blossoming time $^{[10]}$. The mean cosmos inflorescence date for the past 38 years was September 8 . The inflorescence date was September 6 both in the 1970s and in the 2000s. Compared with the starting date of the temperature season, the date was 19 days earlier in the 1970s and 18 days earlier in the $2000 \mathrm{~s}$, and 16 days earlier on the average. Both the cosmos inflorescence date and the starting date of the temperature season did not change, and the Kendall- $\tau$ correlation analysis results showed no coherence.

\subsubsection{Dragonfly}

The dragonfly lives worldwide, and has been designated as the standard fall bug. The starting date of fall was estimated based on the first sighting of the dragonfly. The mean first sighting date of the dragonfly for the past 38 years, June 4, was closer to the mean starting date of summer, June 5, than to the mean starting date of fall in the temperature season, September 24. Although the dragonfly species that was observed was unknown, among the 107 dragonfly species, the first dragonfly sighting date became 21 days later, from May 23 in the 1970s to June 13 in the 2000s. The Kendall$\tau$ correlation analysis results for the two elements showed no coherence for summer or fall.

\subsubsection{Ice}

Ice is the solid state of water. It is formed by the congelation of liquid water, the sublimation of vapor, the compression of accumulated snow, the incursion of water into snow or the congelation of snow. The freezing point of pure water is $0^{\circ} \mathrm{C}$, but it can vary according to the water impurities and ingredients ${ }^{[12]}$. The starting date of winter was estimated from the first sighting of ice. The mean first sighting date of ice for the past 38 years was November 15 . The first sighting date was 
November 16 in the 1970s and almost the same date, November 15 , in the 2000s. Compared with the starting date of the temperature season, the date was 19 days earlier in the 1970s and 24 days earlier in the 2000s, and 22 days earlier on the average. The first ice sighting date became one day early, but the starting date of the temperature season became four days late. The Kendall$\tau$ correlation analysis resulted in a correlation coefficient of 0.37 and a significance level of 0.01 .

Based on the inflorescence and phenomena of living things, the changes in the seasons were -0.183 day/year for the forsythia inflorescence date, -0.061 day/year for the initial cicada sound date, -0.122 day/year for the cosmos inflorescence date, 0.837 day/year for the first dragonfly sighting date, and -0.008 day/year for the first ice sighting date.

Among the biotic season elements, only the dragonfly's change rate increased, which indicates an increasingly late dragonfly observation date. The forsythia inflorescence date ( -0.183 day/year) showed the second greatest change, followed by the cosmos inflorescence date (-0.122 day/year). The least change was seen in the first ice sighting date, which was trivial (-0.008 day/ year). However, all the biotic season elements, except for the dragonfly, had increasingly fast observation dates, and the inflorescence dates of the forsythia and the cosmos, which represent the biotic seasonal elements for spring and fall, significantly changed. The changing trend was stronger in the ice that represents winter than in the cicada that represents summer, which indicates an increase in the summer period and a decrease in the winter period.

\section{Conclusion}

Using the temperature observation data for the past 38 years (1973-2010) from seven ASOSs in the Gwangju and Jeonnam regions, changes in the temperature season and the biotic season (forsythia inflorescence, initial cicada sound, cosmos inflorescence, first dragonfly sighting, and first ice sighting) were analyzed, and the coherence between the temperature season and the biotic season was analyzed using the Kendall- $\tau$ correlation analysis.

The results of this study showed limitations in the classification of the temperature and biotic seasons. Especially in the case of the biotic season that was based on a single life, the points of the first sighting and the initial sound fluctuated so much that the accuracy of the results was not guaranteed. Therefore, the life species have to be selected subject to detailed verification and accurate specifications and to be applied to the meteorological phenomena.

In addition, there are lives in standard biology that can no longer be observed because of environmental pollution and climatic change, which points to a need to protect the existing standard biology. A nationwide temperature and biotic season analysis is required to present a more accurate and systematic season classification standard.

\section{Acknowledgment}

This study was supported by research fund from Chosun University, 2013

\section{References}

[1] J. Freeman, "Science 101: Ecology", HarperCollins, pp. 56-57, 2010, accessed June 2, 2013, http://www. harpercollins.com/browseinside/index.aspx?isbn13= 9780060891336

[2] NIMR, "Climate Change Scenarios Report for the corresponding IPCC the Fifth Assessment Report 2011", pp. 1-111, 2011.

[3] GRMA, "Report of Regional Climate Change Gwangju, Junnam-", pp. 59-60, 2011.

[4] H. W. Park and C. S. Ryu, "The variation patterns of daily over a period of 10 days and precipitation regions of summer precipitation in Korea", J. Korean Earth Science Society, Vol. 26, pp. 417-428, 2005.

[5] C. S. Ryu, H. S. Won, and S. H. Lee, "A study on the influence of aerological observation data assimilation at Honam area on numerical weather prediction", J. Korean Earth Science Society, Vol. 26, pp. 66-77, 2005.

[6] J. T. Hardy, "Climate change: causes, effect, and solutions", Life Science Publishing, pp. 85-99, 2011, accessed June 2, 2013, http://www.kyobobook.co.kr/product/detailViewKor.laf?ejkGb=KOR\& mallGb $=$ KOR\&barcode $=9788961541220$ \&orderClick $=\mathrm{LAV} \& \mathrm{Kc}=$

[7] D. R. Kang, "The characteristics of climate change as the extreme climate unit in Gwangju region", J. Climate Change \& Environment, Vol. 1, pp. 43-49, 2013. 
[8] NIMR, "Understanding Climate Change. 6 Climate Chane of Jeju", pp. 50-51, 2010.

[9] NIMR, "Correspondence of climate change agreement regional climate scenarios utilizing technology development(III)", p.599, 2007.

[10] K. H. Lee, S. E. Hwang, and C. S. Ryu, "A study on the climate change in Homan region", Anal.
Korean Earth Science Society, Vol. 2, pp. 61-65, 2010.

[11] "Doopedia," Doosan Corporation, accessed Aug 1, 2010, http://www.doopedia.co.kr/

[12] "KMA encyclopedia," KMA, accessed Jan 1, 2010, http://web.kma.go.kr/search/kmaSearch.jsp 